\title{
Aneurysmal circumflex coronary artery with fistulous connection to the coronary sinus
}

\author{
Afksendiyos Kalangos, MD, PhD, DSc(Hon), FECTS, ${ }^{a}$ \\ Saziye Karaca, $\mathrm{MD}^{\text {a }}$ Mustafa Cikirikcioglu, $\mathrm{MD}, \mathrm{PhD}^{\text {a }}$ \\ Dominique Vala, MD, ${ }^{\mathrm{a}}$ and Dominique Didier, MD, ${ }^{\mathrm{b}}$ \\ Geneva, Switzerland
}

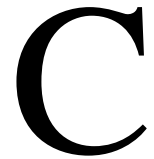

ircumflex artery fistula to the coronary sinus is a rare clinical condition, with only 4 cases previously reported in the literature. ${ }^{1-4}$ We describe another case of congenital circumflex artery aneurysm draining into the coronary sinus in an adult woman.

In this 66-year-old female patient, a pathologic cardiac murmur had been diagnosed 10 years previously, and further cardiac workup showed the presence of a circumflex artery fistula to the coronary sinus without any aneurysm formation. The patient refused the interventional closure of this fistula because she was asymptomatic. During these 10 years, she had progressive dyspnea on exertion that was further complicated by the onset of permanent atrial fibrillation. She finally agreed to an operation, but before surgical intervention, she was urgently admitted to the hospital for congestive heart failure with pulmonary edema. Workup consisted of echocardiography, cardiac catheterization, multislice cardiac gated computed tomography (CT), and coronary angiography. Echocardiography showed dilatation of both atria and ventricles with severe tricuspid insufficiency and mild-to-moderate mitral insufficiency. At cardiac catheterization, pulmonary artery pressure was 55/12 mm Hg (mean, $30 \mathrm{~mm} \mathrm{Hg}$ ), pulmonary/systemic flow ratio (Qp/Qs) was 2.7, and left ventricular ejection fraction was estimated at $45 \%$. Coronary angiography revealed normal left anterior descending and right coronary arteries. The circumflex territory was occupied by a large aneurysm, from which 2 rudimentary obtuse marginal branches originated (Figure 1, A). This aneurysm terminated in a large fistulous connection into the coronary sinus. CT imaging confirmed a circumflex artery aneurysm of $5 \times 4 \mathrm{~cm}$ and revealed the presence of 2 fistulas between the distal part of this aneurysm and the coronary sinus (Figure 1, $B$ and $C)$. Surgical intervention was performed with extracorporeal circulation during mild hypothermia, and antegrade cardioplegia was first applied while occluding the ostium of the coronary sinus with a Fogarty balloon catheter through a right atriotomy. Cardioplegic

\footnotetext{
From the Clinic for Cardiovascular Surgery ${ }^{\mathrm{a}}$ and Department of Radiology, ${ }^{\text {b }}$ University Hospital of Geneva, Geneva, Switzerland.

Received for publication Jan 19, 2005; accepted for publication Feb 8, 2005 .

Address for reprints: Afksendiyos Kalangos, MD, PhD, FECTS, Clinic for Cardiovascular Surgery, University Hospital of Geneva, 24, rue Micheli-duCrest 1211, Geneva, Switzerland (E-mail: afksendyios.kalangos@hcuge.ch).

J Thorac Cardiovasc Surg 2005;130:580-1

$0022-5223 / \$ 30.00$

Copyright (C) 2005 by The American Association for Thoracic Surgery doi:10.1016/j.jtcvs.2005.02.045
}
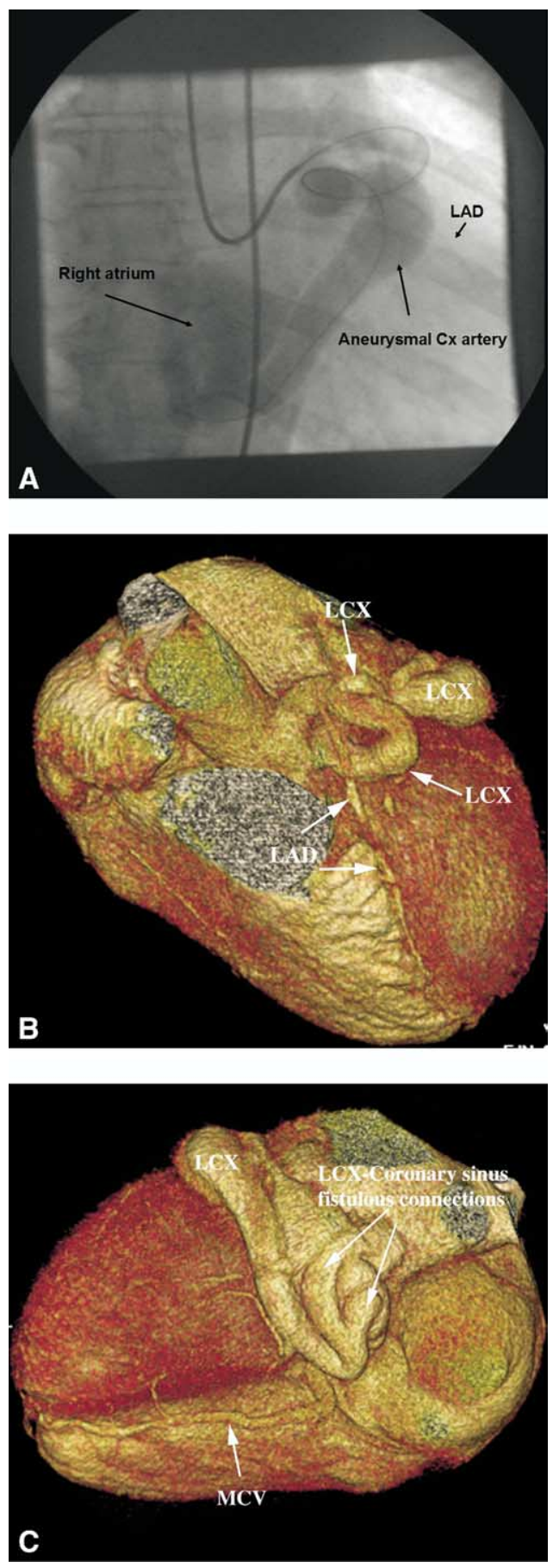

Figure 1. A, Coronary angiogram showing the tortuous aneurysmal circumflex artery opacifying the right atrium. B and C, Multislice cardiac gated CT showing the left anterior descending artery and the tortuous proximal segment of the circumflex artery connecting to the coronary sinus with 2 fistulas. $L A D$, Left anterior descending artery; $C x$, circumflex; $L C X$, left circumflex artery; MCV, middle cardiac vein. 


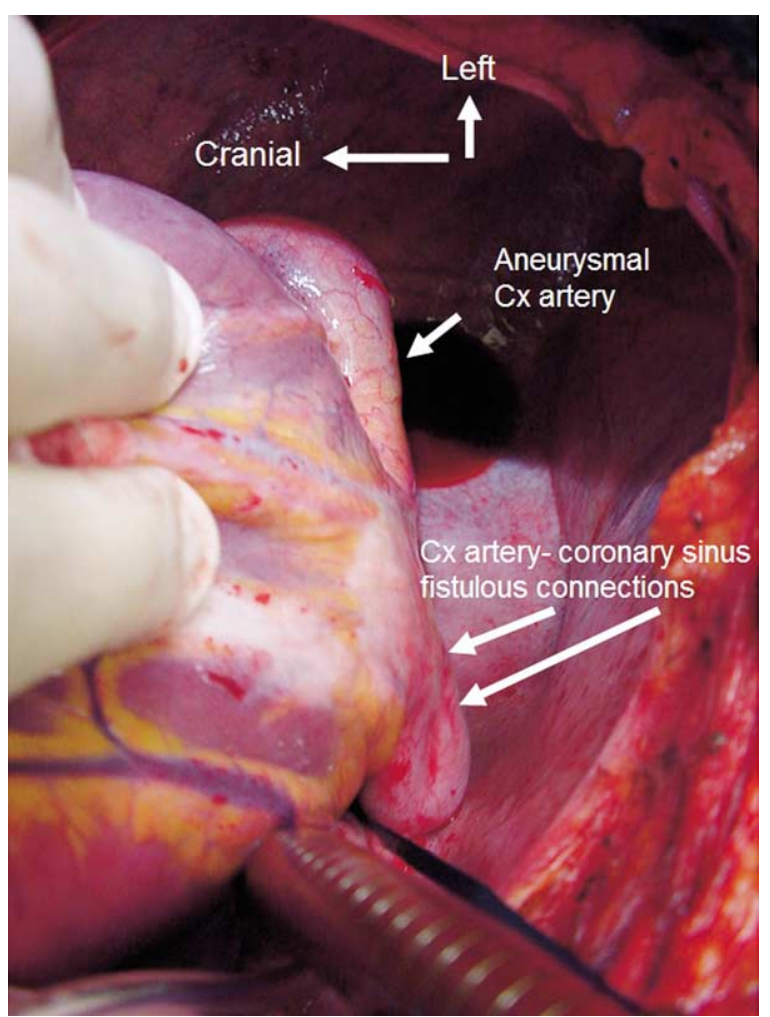

Figure 2. Operative photograph showing the distal segment of the circumflex $(C x)$ artery aneurysm with the fistulous connections to the coronary sinus.

solution was then delivered retrogradely to optimize myocardial protection of the aneurysmal circumflex territory. The aneurysmal circumflex artery was ligated at its origin and then opened over its entire course, and the 2 fistulous connections into the coronary sinus were obliterated with a running 4-0 suture (Figure 2). During exploration, we remarked that the 2 rudimentary obtuse branches originated from the coronary sinus behind the aneurysmal circumflex artery and were perfused by the blood flow provided by the circumflex artery to the coronary sinus through the fistulous connections. The diameters of these branches were smaller than 0.5 $\mathrm{mm}$ and judged to be unbypassable. Concomitant De Vega tricuspid annuloplasty was subsequently performed to treat the severe functional tricuspid insufficiency. The postoperative course was complicated by a lateral myocardial infarction, which was hemodynamically well tolerated. On follow-up at 12 months, the patient is in New York Heart Association class II.

As in our case, coronary fistulas are usually well tolerated and diagnosed it adults, when symptoms of congestive heart failure caused by longstanding volume overload appear. The onset of atrial fibrillation, ventricular arrhythmia, acute myocardial infarction, or ischemia can precipitate the appearance of these symptoms. Antegrade microcoil embolization of the fistulous connection by catheterization is indicated to prevent progressive congestive heart failure, endocarditis, and coronary aneurysm formation with rupture or embolization. ${ }^{2,4}$ Surgical closure is especially indicated in cases of coronary artery aneurysm or in cases in which a concomitant operation is required for an associated lesion, such as for the severe tricuspid insufficiency in our case.

\section{References}

1. Chamberlain MH, Henry R, Brann S, Angelini GD. Surgical management of a gigantic circumflex artery aneurysm with fistulous connection to the coronary sinus. Eur J Cardiothorac Surg. 2001;20:1255-7.

2. Bauriedel G, Skowasch D, Lauck G, Schmitz C, Breuer J, Luderitz B. Micro-coil embolization of a fistula of the circumflex ramus in the coronary sinus: case report, differential therapy and review of the literature. Z Kardiol. 2002;91:261-6.

3. Dogan A, Ozaydin M, Altinbas A, Gedikli O. A giant aneurysm of the circumflex artery with fistulous connection to the coronary sinus: a case report. Int J Cardiovasc Imaging. 2003;19:5-8.

4. Perry SB, Rome J, Keane JF, Baim DS, Lock JE. Transcatheter closure of coronary-artery fistulas. J Am Coll Cardiol. 1992;20:205-9. 